\title{
ENERGY EFFICIENT SECURE HYBRID BIO INSPIRED CONGESTION CONTROL MECHANISM IN WIRELESS SENSOR NETWORK
}

\author{
Suchitha.S \\ Research Scholar, Bharathiar University \\ Coimbatore, TamilNadu, India \\ Dr.B.G.Prasanthi \\ Associate Professor, Computer Science Department \\ St.Joseph's College, Bangalore, India
}

\begin{abstract}
In wireless sensor networks, congestion control is a major issue. When incoming packets increase beyond the actual ability of the network, otherwise node, it leads to congestion in the network. Network congestion can reduce bandwidth, increase network delay, as well as increase packet loss along with sensor power loss. Therefore, better methods are needed to deal with congestion. There is a need to understand congestion as well as supervise congestion resources in the wireless network to improve network performance. In recent years, various methods for recognizing and preventing congestion have been introduced. To address some of these issues, biodiversity monitoring system called LionFuzzyBee is proposed. The LionFuzzyBee is compared to a lion and a bee methods by a variety of metrics, for instance packet delivery ratio, detection accuracy, average throughput along with energy consumption etc . Evaluation outputs demonstrated that the LionFuzzyBee provides enhanced outputs than the Lion as well as Bee algorithm.
\end{abstract}

Keywords: Wireless sensor networks (WSNs); Congestion Control; Bee Optimization; Lion Optimization and LionFuzzyBee.

\section{Introduction}

WSN on a wireless sensor network is a network of systems that can be able to densely located in an aggressive as well as unreachable environment to sense this atmosphere along with the collected information through high accuracy. It incorporates sensors, calculations, as well as communication into a tiny system called a sensor node [1-4]. WSN consists of thousands of device nodes. The sensor unit consists of single or additional sensors associated with the application, the vital device being a microwave, a battery memory relay [5,6]. A collection of sensors connected via a wireless storage device to perform distribution recognition tasks. The major job of the sensor is to know the data in the surroundings, process this data as well as transmit the information to the sinking unit. There are numerous issues in WSN [7-10].

When a node's output information goes to a single base station in the WSN for a long time, there is a risk of network shutdown. When the recipient node receives a packet of data at a level higher than its ability, extra information needs to be stored in the cache [11]. Because of insufficient free space, the buffer is occupied as well as the information packet must be freed accordingly to congestion [12]. Congestion is a major problem in WSN that will affect the quality parameter. Congestion occurs at what time the speed of a packet entering a particular hub is superior than the amount of packets issued. Overloading produces packet failure as well as reduces reply time [11].

Congestion in the WSN will lead to degradation of network processes, for instance reduced bandwidth, distribution ratios, enlarged latency as well as power consumption per packet . It is important to pay attention to congestion problem in the network to provide the necessary transmit rate for WSN as well as extend network life . Congestion in the WSN occur at the node stage or connection stage [14,36]. At what time the packet reached value is superior than the packet service speed, after that node stage congestion will happen. This type of congestion causes packet failure as well as decrease network life. When multiple sensor centers in such a 
range try to transmit information simultaneously, there will be a serious breakdown of the connection. This type of collision will interfere with network usage and full outcomes.

Hence, for decades, congestion management has turn out to be an lively region of research, but usually fewer attention is given to congestion management in the evolving parts of the WSN. To send data to WSN, first create a path using any routing protocol. Large amount of packets are transmitted via network at a time, which produce network congestion for a variety of reasons, such as cache overload, low network capacity, and so on. In the WSN, several congestion control designs have been created through researchers. Current approaches to congestion management have few problems, for instance future traffic administration, regardless of the evaluation of alternative road traffic [13]. From the source sensor node to the sink, priority is given to the number of hops instead of the actual packet delay $[15,16]$. In addition, the sharing of transfer loads on crowded along with alternating paths is not appropriately addressed. To overcome these problems, this work suggests an inspired bio algorithm called LionFuzzyBee.

The major offerings of this work are summarised as follows:

(i) To reduce congestion in WSN using LionFuzzyBee optimization approach.

(ii) To improve the quality of WSN by reducing packet loss.

(iii) To reduce the execution time of optimization approach by combining Lion, and Bee approaches.

(iv)To boost the life time of WSN by increasing the energy efficiency.

(v) To increase the scalability of WSN.

A secondary contribution is to implement the congestion control techniques presented in the WSN and to analyze the implementation by comparing it with existing methods.

The manuscript of this document is prepared as follows: Section 2 talks about some current related work. Section 3 offers a detailed explanation of the presented architecture. Section 4 presents experimental results that include general operating results from LionFuzzyBee, performance comparisons and other previous methods. Section 5 presents the Conclusion.

\section{Related Work}

Various techniques have been developed for congestion management as follows: [20] The authors propose a congestion avoidance protocol called machine learning cloning (LACAS) based on machine learning. The author called it an automated learning process and then used it to improve the next procedure consistently over time. Depending on the motion stack in the system, the broadcasting speed may be changeable. In this way, to prevent congestion, the training machine restricts the flow of data at all nodes. In this procedure, multiple packets transferred to the intermediate node will be used to the extent that possible to limit the intermediate node limit and reduce packet loss.

Amir Hossein, et al. [21] presented the Bat schemes for a variety of best value selection tasks. They presented mathematical schemes to resolve main functions with match up to different metrics of the bat algorithms with target functions. Bat algorithms try to attain the greatest universal answer subsequent to replicate the mathematical structures can be employed for a variety of troubles, Antonio M. Ortiz et al. [22] presented routing protocol for dense networks. Logical methods are used to determine the best path for information broadcast from source to destination. Paths can be decided with mathematical schemes obtained by means of ambiguous logical algorithms in addition to show improved outputs compared to preceding methods according to delay with package distribution ratio.

Alihodzic, et al. [23] presented the use of programmatic solutions in image processing software. They developed a model that was employed to determine the level of the image through the procedure. In their schemes, they considered several levels to define the binary image of a specified image . This method can be applied to wireless sensor networks with arranging different operating stages for the nodes. Salvador Climent et al. [24] presented the universal structural design of the submarine network with its submerged surface. Maintained by comprehensive data of each detector, DBR (Depth Based Routing) transmits packaged data to the water area. The DBR packet has a field that records the last sender 's depth information and is modified by individual clusters.

Seokhoon Yoon et al. [25] presented the use of AURP (An auv-aided underwater routing protocol) to solve the problem of small proportion of packet transmission size as well as large amount of power utilization in the underwater acoustic sensor network. To understand the link between the size of the information and the low power consumption, AURP uses the control quality of several autonomous submarines that are similar to the same acoustic broadcasting network. Sheng-Shih Wang et al. [26] presented a connection-based scheme to grouping in a wireless sensor network. Connections are accumulated in the routing table of all node along with packets are transmitted through these connections, which keeps high amount of energy employed to define the connection. This protocol defines the common MAC protocol as well as intends to increase the network life by distributing the remaining power of the sensor nodes. 
Selim Yilmaz et al. [27] presented mathematical methods to solve the problem of creating multiple obstacles. This type of problem requires continuous optimization, finding the best local and other solutions. The Bat algorithm is used to solve this type of problem. Miao Zhao et al. [28] developed a wireless battery network that uses rechargeable batteries to operate the node. These transportable nodes can be located in the network as well as can be employed to calculate the status of the network along with employed for the computation of nodes in the network. Xin-She Yang et al. [29] presented algorithms derived from study techniques employed through bats to find paths or food. They use sonar waves In this work, they calculated a mathematical replica for the similar that thinks each and every one of constraints determining the best universal result.

Miguel Garcia et al. [30] presented a cooperative wireless sensor network in which clusters were created through nodes and data dispensation was performed with group heads of all cluster. The group head should have the optimal power arrangement and should have a similar number of remaining nodes in the network. This setting assists to improve the ratio of packet distribution as packets go behind multiple jumps to the base station.

In [31] the authors presented a technique to avoid congestion. Three mechanisms are here for domineering congestion in the sensor network, which is the buffer state of each receiver to detect congestion, bounce back pressure, and control the speed of multiple sources in a closed loop. Congestion recognition is one that is aware of the buffer tenancy of each node as well as through approximates the channel load conditions in excess of a broad period of time. When an overload is detected in the system, the nodes at this point transmit loops to adjacent nodes to reduce their transmission rate. If the congestion in the network remains constant, then we use a mechanism to control the speed of the closing cycle. This method maintains end-to-end response from source to sink node via acknowledgement packet.

When receiving the ACK packet, the output changes its broadcast speed, lets the source does not receive the broadcast packet, after that it regulates the broadcast speed manually. CODA protocol helps to decrease congestion, except it cannot get rid of it, so it does not guarantee justice in the network. In [32] the authors propose an optimization technique called distribution model compression. To use WSN in a power proficient way, we occasionally require shrinking the network to a larger one so that network life can be extended. Distributed compression model is a single method to employ. The choice to compress as well as send it to the subsequently node is taken through the node in the network. Therefore, the small precedence package to be delivered will be condensed along with merely the elevated precedence package will attain the destination earlier. Pressure is an important part of extending the life of the scheme as well as it should be used as division of the path by means of the ultimate ambition of reducing packet failure.

Wang et al. [33] presented a disseminated Max-Min Fairness schemes designed to reduce congestion. It is derived from the ALOHA, so that the node employs information about the total to calculate its possibility. Once detecting the possibility rate, the speed of the signal is determined as well as it may differ plus be able to distorted along with employed through ecological situation. This way the congestion will be diminished. To achieve greatest plus smallest amount fairness in this case, decentralization schemes are employed. In TARA, at what time a node is documented as having a channel load congestion as well as the buffer tenancy of that node is quickly calculated, this node is measured a node of the difficulty region. In addition, using this problem area, the motion of the nodes is forwarded through the assumed nodes to the spot in a transformative manner and reaches the unifying nodes where the flows merge. Once the blockage is cleared, the system discards the use of diversion [34].

At [35] the authors anticipated a procedure that employs buffers along with caches to look for congestion in the sensor network. When congestion happens on the network, it employs a stretchable alignment program that chooses which small precedence package will be cleared in addition to which one will be sent subsequently. It employs two sub-rows, one for privately created packages and the other for transitional action packages. Queued packets are organized from the source point of view at what time is momentary crowd. As a source, a package is sent from a impermanent one, furthermore a locally generated package is transmitted using a rotation algorithm. Transient along with congestion are dissimilar as well as are treated differently. The ECODA method does not require a clear ACK from the sink. Using this method, nodes can be recognized by blocking with arranging dynamic and accurate source transmissions.

\section{Materials and Methods}

Bio based congestion control systems can be made up of systems that can configure hardware or software as well as electronic devices that mimic the way data is processed as well as bio systems are addressed. At what time we seem at nature, a lot of different behaviors are experienced. The majority of species exhibit social behavior. In few species, there is a leading individual who guide all the members of the group. It is similar with lions, deers. Nevertheless, there are additional species that exist in clusters without a main leader.

For those animals, individuals have a prearranged attitude that permits them to pass by their surroundings without guidance, for instance birds, bees, ants. 
In this second kind of performance, animals do not know the cluster as well as the surroundings wherein they shift. As an alternative, they travel around the surroundings distribution data with their neighboring affiliates. This easy communication among individuals complicates cluster performance. These performances are generally the consequence of several existence of natural development. But a bio-inspired single congestion management system has some disadvantages, such as a lot of working time to find the cost of high memory optimization, suitable only for a small area, does not provide the best coverage problems, and depends only on global or local search engine. To overcome this shortcoming, a hybrid bio congestion control system was developed in this work.

The presented hybrid bio-inspired Congestion control system incorporates Bee and Lion algorithm. These algorithms and the presented LionFuzzyBee algorithm are briefly discussed in the next paragraphs.

\subsection{Artificial Bee Colony Algorithm}

ABC was first introduced by Dervis Karaboga [17], inspired by the collective behavior of honey bees with improved performance in function enhancement. Colonization of insects in society can be seen as a powerful system that collects information from the environment and modifies its behavior. There are main components of the ABC optimization model for food source selection that lead to the emergence of collective intellectuals of bee genes. There are two attitudes, giving up and gathering food sources.

\subsection{Lion Optimization Algorithm}

The Lion Optimization (LOA) algorithm presented by Wang [19] and Rajakamar [18] is based on the social behaviour of lions. This is a metaheuristic algorithm which is part of the stochastic optimization. These algorithms can create different solutions to problems in each implementation .

\subsection{Proposed Methodology}

The LionFuzzyBee defines bio-inspired algorithms in WSN that use location information to manage congestion. New cluster-based WSNs are also used in this work to reduce packet loss and save energy. It can find the best or closest reliable path at what time the network has nodes with dissimilar broadcast ranges with the least power consumption. The general architecture of LionFuzzyBee is shown in Fig.1.

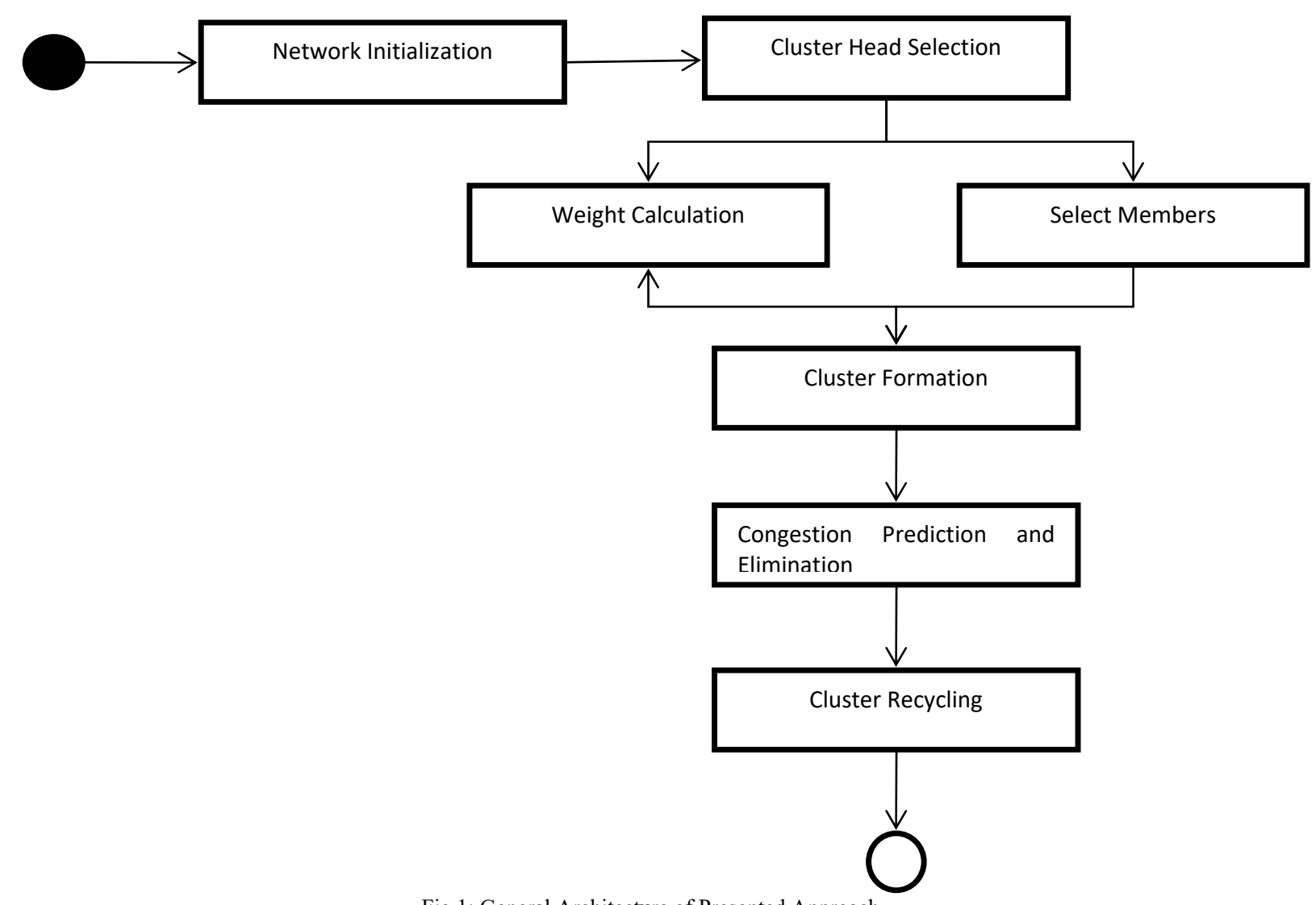

Fig 1: General Architecture of Presented Approach. 
LionFuzzyBee responds thus finding the path only if a packet of data is to be sent from the source node, say Q to the destination node, say T. In the next section, this article describes the key steps of the new WSN approach based on clusters that play a key role in this algorithm. The steps are

1. Network Initialization

2. Region Splitting

3. Initial Cluster Head Selection

4. Cluster Formation, Building Phase

5. Congestion Prediction and Elimination using LionFuzzyBee

6. Cluster Recycling

\subsubsection{Network Initialization}

Start networking with nodes and mobile sink (LionBee). First, mobile devices are located . In the initial stage to move the mobile sink near the cluster each group of clusters contains mobile sinks. The process of network start-up is shown in Fig. 2

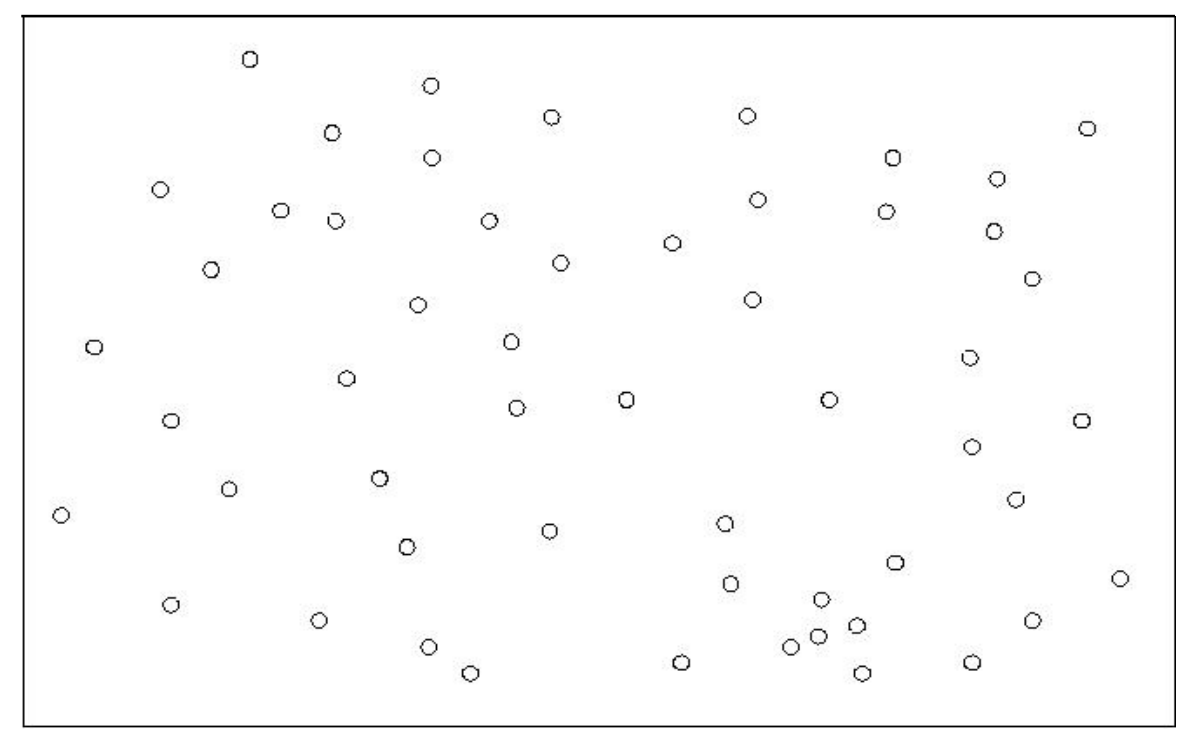

Fig 2: Network Initialization of Presented Cluster based WSN

\subsubsection{Region Splitting}

Considering the direction node $\mathrm{T}$ along with the $\mathrm{G}$ network graph, all nodes are splitted with its neighbors taken with 4 sectors named S1, S2, S3, and S4. Consider the part of the line between Q to T. For HS neighbors, the $\theta H$ angle is described as the angle between sectors. The node $N$ belongs to $S 1$ if $\theta N \leq \pi / 4, S 2$ if $\pi / 4<\theta N<3 \pi / 4$, and $\mathrm{RS}$ if $3 \pi / 4 \leq \theta \mathrm{N}$, this is shown in Fig.3. 


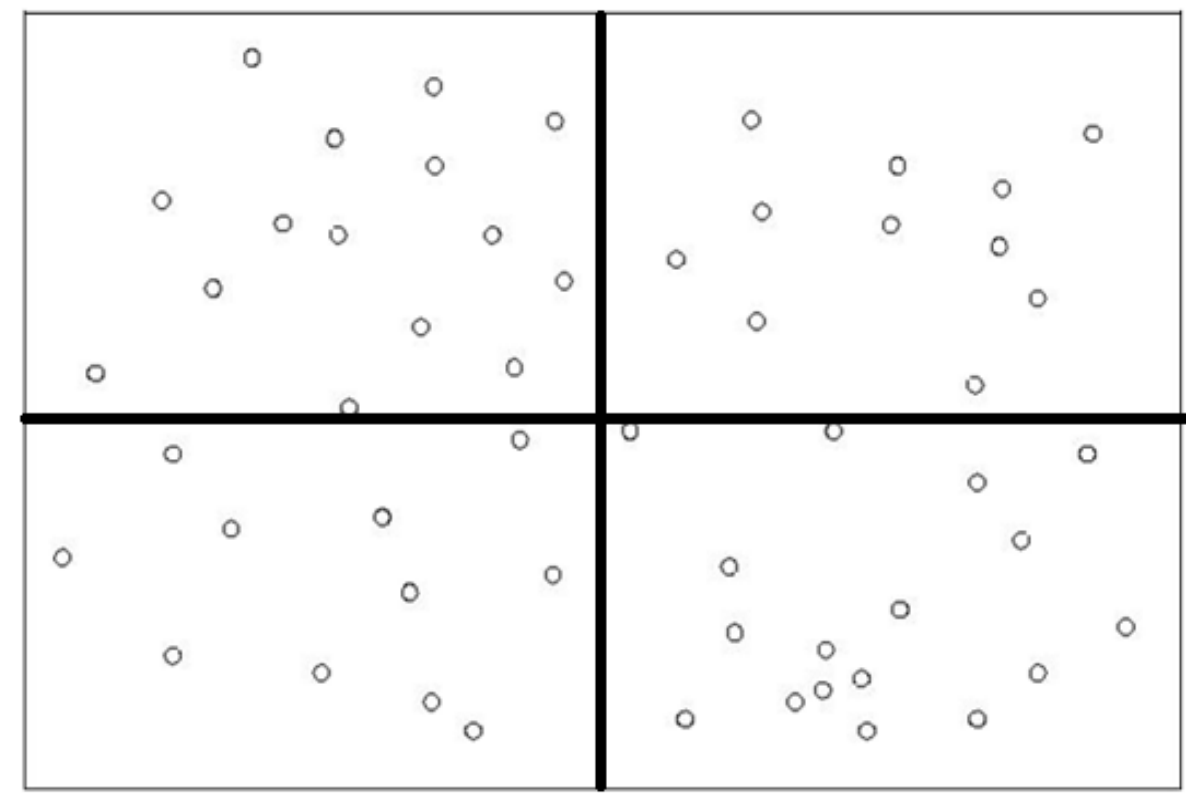

Fig 3: Region Splitting of Presented Cluster based WSN

\subsubsection{Initial Cluster Head Selection}

In this phase, select a cluster head for each group. To calculate the weight, select the cluster head. The weight calculation is based on the distance between the unit and the sink and the power of the unit. But initially all nodes has the same energy. Each head of the cluster has a neighbourhood group. This is shown in Fig.4.

The presented method follows a distributed method to create a hierarchical configuration in a selforganizing regime exclusive of vital rule. The selected method picks arbitrary nodes to activate the cluster procedure in advance. Active node Ut compute the relative density as well as its space from the base station to decide the circumference of cluster $\mathrm{k}$ transient (1) as well as fetching the transient head of the cluster.

$$
k=\text { floor }\left[\beta D\left(U_{t}\right) / D_{k}\left(U_{t}\right)\right]
$$

Where $D_{(\mathrm{w})}$ is the space from the base station of $\mathrm{u}, D_{k}(\mathrm{u})$ is the connection thickness of the node $\mathrm{u}, \beta$ is the parameter of the device defined by the specific WSN application, and the other part is the calculation. Round. $D_{(w)}$ can be deliberated as follows.

$$
D(u)=10 \frac{|R S S I-A|}{10 . n}
$$

Where RSSI is a power indicator and A is a force of power from the station.

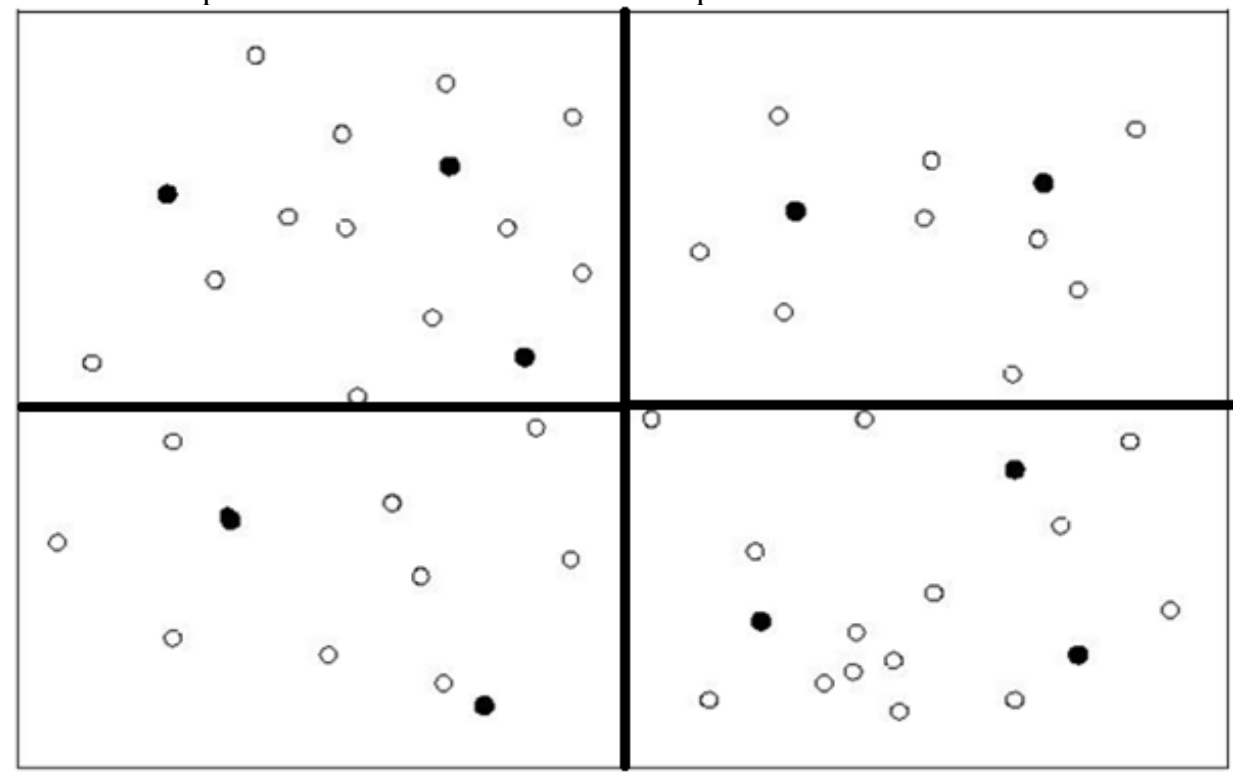

Fig 4: Initial Cluster Head Selection of Presented Cluster based WSN 


\subsubsection{Cluster Formation, Building Phase}

The presented method determines the group range. The amount of group nodes cannot be exceeded to keep away from the formation of huge groups, which will lead to additional costs as well as decrease network life. At once the group master obtains a join message dispatched through the normal node, it compares the range of the group to the recent member reception level as well as updates the number of group nodes if the range is less than the threshold otherwise rejects this demand. If the discarded node already has a $\mathrm{CH}$, the grouping procedure will stop. If not, it discovers a new suitable group to connect.

Every member node of the group preserves a group data table that stores CHID, CH, MID along with other data. If the node obtains a packet transmission in the job, it will revise its group data table accordingly. For instance, the $\mathrm{CH}$ check node in the recently obtained packet, if the $\mathrm{CH}$ is minor, it will update the $\mathrm{CH}$ value in the table through the MID update. Specifically, it finds a fast route to the group head as well as identifies the recent MID as its node. There is merely one CHID entry in a normal node since it fits in to a group head, other than partly cover nodes have numerous CHID access for dissimilar groups. The presented algorithm keeps away from the scheme of a permanent group with intermittent substitute to stable the power utilization of the node. This is shown in Fig.5.

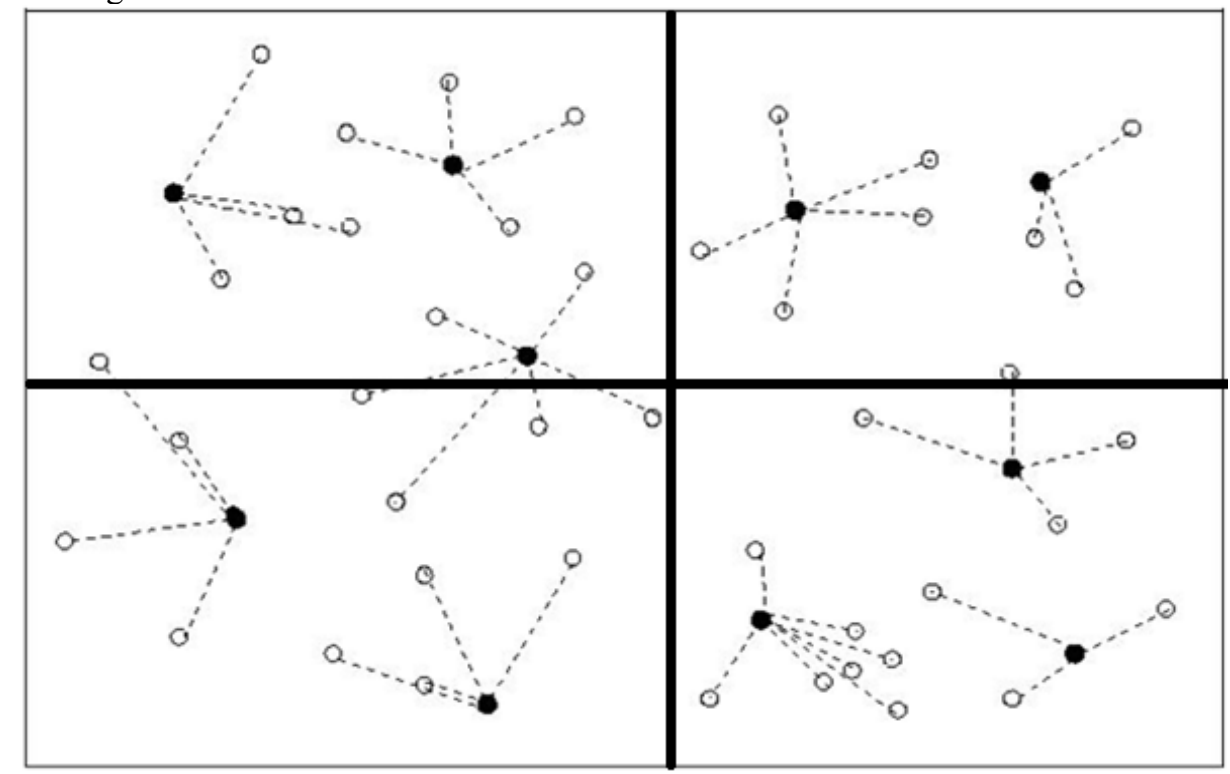

Fig 5: Cluster Building Phase of Presented Cluster based WSN

After the clusters are formed, the cluster heads are connected together to produce a reliable power method. This is shown in Fig.6.

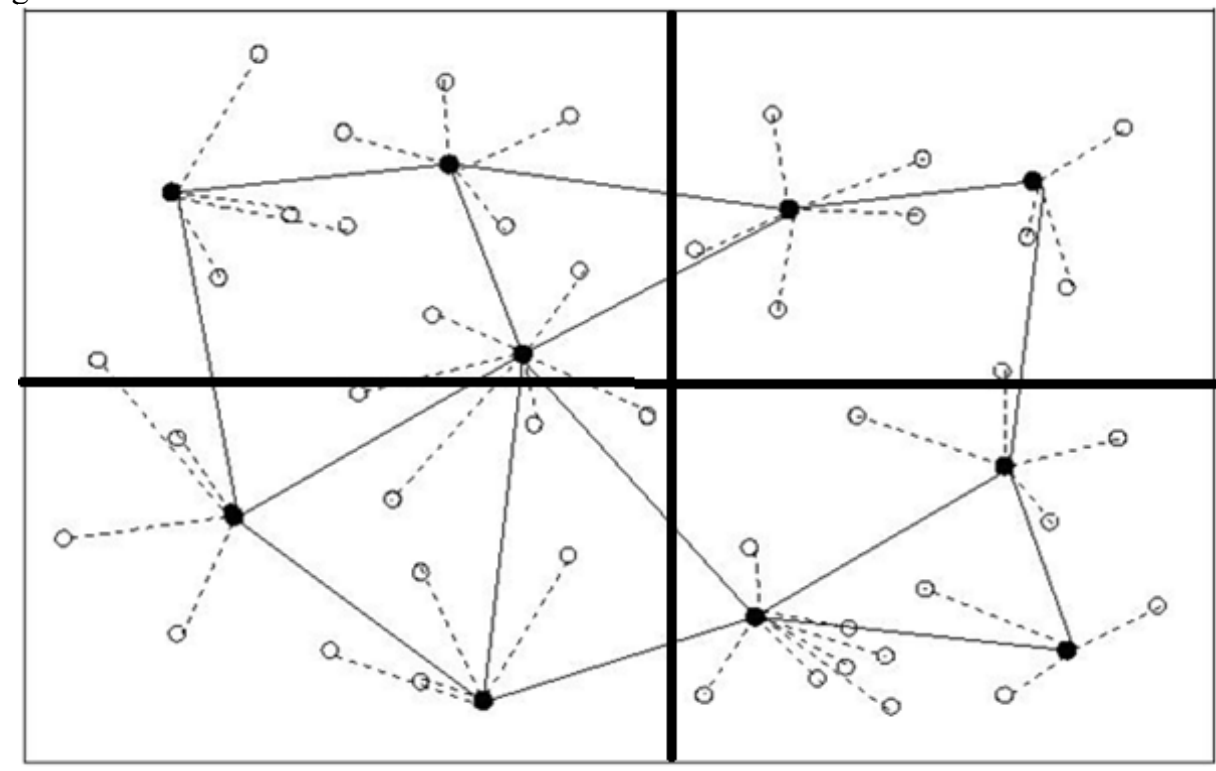

Fig 6: Cluster Connection Phase of Presented Cluster based WSN 


\subsubsection{Congestion Prediction and Elimination using LionFuzzyBee}

In this work, LionFuzzyBee Approch is used for prediction and controlling congestion. This is a combination of lion and bee optimization. The LionFuzzyBee method has the following advantages.

(i) They can adapt to environmental changes.

(ii) It demonstrates power and confrontation to damage through internal or external factors.

(iii) Allows the application of complex situations and behaviors within limited basic limits.

(iv) These systems can be learned and developed when new conditions arise.

(v) They can handle restricted resources.

(vi) The group of nodes that execute these schemes can be self-organized in a completely disseminated method, effectively integrated with LionFuzzyBee.

LionFuzzyBee optimization technique can now be defined as the functions described in Equations $3,4,5$, and 6 , which are the most universal approximations for subsequent optimization problems:

LionFuzzyBee $(\mathrm{Y}, \mathrm{K}, \mathrm{L})$

where $\mathrm{Y}$ is the function that randomly produce the first solution, $\mathrm{K}$ prepares the original values offers through the function $\mathrm{Y}$ as well as returns $\mathrm{L}$ at what time the final decisive factor is fulfilled. The functions $\mathrm{Y}, \mathrm{K}$ and $\mathrm{L}$ are termed as follows:

$$
\begin{aligned}
& \partial \vec{A}\left\{\mathrm{P}_{\text {Bee }}, \mathrm{P}_{\text {ов }}, \mathrm{P}_{\text {Beelion }}, \mathrm{P}_{\text {овц }}\right\} \\
& \left\{\mathrm{P}_{\text {Bee, }}, \mathrm{P}_{\text {Beelion }}\right\} \vec{A}\left\{\mathrm{P}_{\text {Bee, }} \mathrm{P}_{\text {Beelion }}\right\} \\
& \left\{\mathrm{P}_{\text {Bee, }}, \mathrm{P}_{\text {Beelion }}\right\} \vec{A}\{\text { true,false }\}
\end{aligned}
$$

Where PBee is the position matrix of the bee, PBeelion eports the position of the beelions, Ров is the corresponding bee and POBL is the appropriateness of the beelions.

The simulated technical code is described as follows:

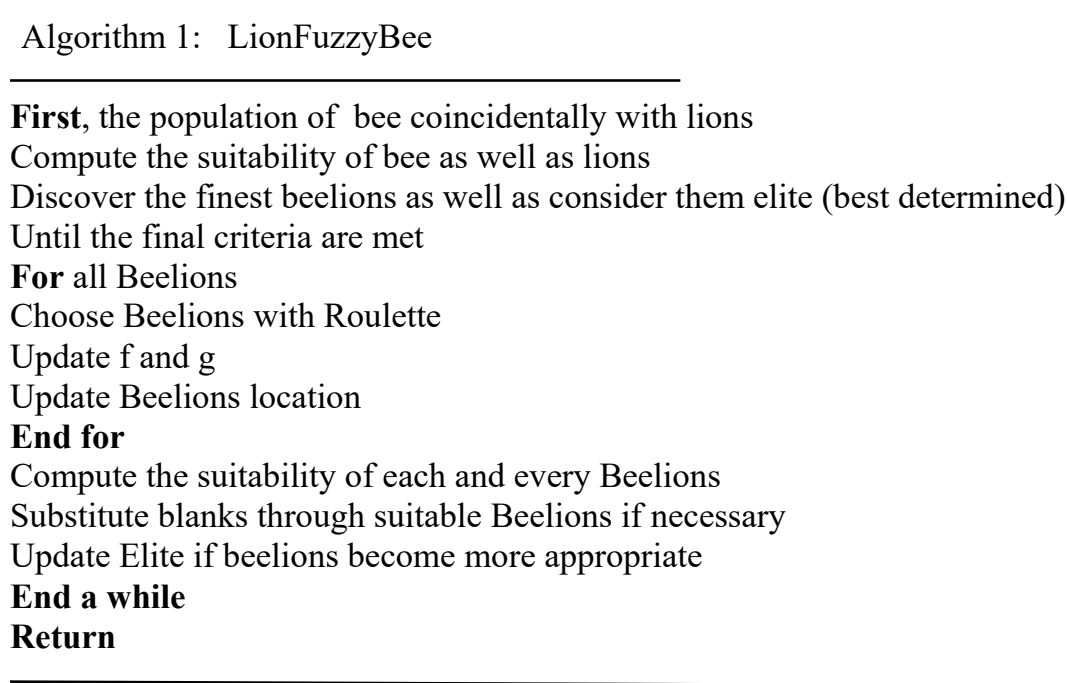

By LionFuzzyBee method, the initialization of lions and bees was randomly started using the $\mathrm{E}$ function. When repeated, the $\mathrm{F}$ function recalls the position of each bee relative to the fitness selected by the roulette operator along with the elite. Restrictions on initial positions are in proportion to the number of repetitions. A place for the update is possible through two random walks around the current and selected elites. Whenever all the bees fly by accident, they are calculated using the fitness function. If one of the bees is better than the other, their location is measured at the new location for the bell in response. The greatest beelions are combined with the greatest value found in updated (elite) and are replaced if necessary. These steps are the same until the function returns incorrectly. 
a) Congestion prediction using LionFuzzyBee

LionFuzzyBee predicts the congestion behaviour earlier by observing the packet flow and network traffic between the cluster head and cluster member.Among several network traffic parameters, LionFuzzyBee selects queue length as the main parameter and its updates the queue length parameter in routing table also. By using this parameter it predicts the congestion behaviour earlier. If cluster member wants to send the packet to cluster head, first LionFuzzyBee calculates the fitness of cluster head (source) using the queue length. If the fitness is higher than the CCR (Congestion Control Rate), cluster head is ready to accept new packet because congestion will not appear and CPF (Congestion Prediction Flag) is 0. Otherwise cluster head is busy and its not ready to accept new packet because congestion will appear and CPF is 1 . Finally we have to eliminate by using LionFuzzyBee. The Algorithm for Congestion prediction using LionFuzzyBee is shown in Algorithm 2.

Algorithm 2: Congestion prediction using LionFuzzyBee

Cluster Member (S) initiate packet P to send to Cluster Head (D)

Get Fitness Value $(\mathrm{F})$ by running Algorithm 1

if $\mathrm{F}>\mathrm{CCR}$ then

$\mathrm{CPR}=0$

Transfer P to D

else

$\mathrm{CPR}=1$

endif

Execute Algorithm 3 to eliminate Congestion

b) Congestion Elimination using LionFuzzyBee

If the algorithm 2 get 1 for CPR, the congestion will appear so it is to be eliminated by LionFuzzyBee.It calculate the best optimum solution (another route node) which has the sufficient queue length to transmit the packet $\mathrm{P}$ to avoid congestion using algorithm 1. By following this procedure the congestion will be controlled in very efficient manner and also the energy utilization and packet loss will be greatly reduced. The Algorithm for Congestion elimination using LionFuzzyBee is shown in Algorithm 3.

Algorithm 3: Congestion Elimination using LionFuzzyBee

\section{if $\mathrm{CPR}==1$ then}

Execute Algorithm 1

get best optimum solution and the optimum route node is $\mathrm{RN}$

Generate route by using RN

endif

\subsubsection{Cluster Recycling}

The cluster is constant for some time awaiting the re-selection procedure of the $\mathrm{CH}$ is prompted in $\mathrm{T}(\mathrm{k})$. $\mathrm{CH}$ collects the weight of each and every one member nodes and then pick the node which have the maximum weight as $\mathrm{CH}$. The weight calculated for the above steps. Distance between sink unit and power. The initial power step is the same but after data transfer more power is used. So calculate the remaining energy.

This reduces communication costs. Re-selection of cluster heads occurs in old clusters, so provisional $\mathrm{CH}$ emissions along with equivalent reply of k-hops neighbors are not necessary. Consistent with the standard cost of group transmission can be calculated. 


\section{Experimental Results}

\subsection{Simulation Environment} Table 1.

The presented system was implemented through NS2. In the simulation, several parameters are listed in

\begin{tabular}{|l|l|}
\hline \multicolumn{1}{|c|}{ PARAMETERS } & \multicolumn{1}{c|}{ MEASUREMENTS } \\
\hline Number of Nodes & 50 \\
\hline Area Size & $1000 \mathrm{~m}$ x 1000m \\
\hline Target Size & {$[500,500] \mathrm{x}[500,500]$} \\
\hline Simulation Duration & 900 seconds \\
\hline No of Attacker nodes & 5 \\
\hline Queue Limit & 20 \\
\hline Queue Size & 100 \\
\hline Packet Size & 552 Bytes \\
\hline Packet Interval & 2 \\
\hline Communication Range & 30 m \\
\hline Buffer Size & 20 packets \\
\hline Percentage of Attacker Node & $5 \%$ node \\
\hline Traffic Pattern & Constant Bit Rate \\
\hline
\end{tabular}

Table 1. Simulation Parameters \& its Measurements

\subsection{Performance Metric}

\subsubsection{Packet Delivery Ratio (PDR)}

It determines the total number of packages sent from the available packages.

$\mathrm{SP}=\frac{\text { Total no of dellvened paskets }}{\text { Totel no of availlable paskets }}$

\subsubsection{Average Throughput (AT)} network.

It measures as the average proportion of successful messages transmitted through a communication

\section{AT $=\frac{\text { Total no of Successfully rec elved packets }}{\text { Tol }}$ Total no of twansmit Paskets}

\subsubsection{Detection Accuracy}


It measures the proximity of the measurement between the original malicious node and the malicious node detected by the presented method.

Accuracy $=\frac{\text { Wo of Conectly Detected Malisious:Node }}{\text { Total no of Walici ous:Wode }}$

\subsubsection{Energy Consumption}

This is the average power used by the nodes in the network. It decreases with the attack of the nodes when the transmission packet between source and destination is dropped, resulting in less transmission between nodes.

\subsection{Experimental Analysis}

Packet delivery ratio is one of important parameter for identifying congestion because it counts the number of transmitted packet. The proposed LionFuzzyBee based congestion control approach's packet delivery rate is compared with Lion and Bee based congestion control approaches through varying the number of nodes from 10 to 50 and the result is illustrated in Fig.7.

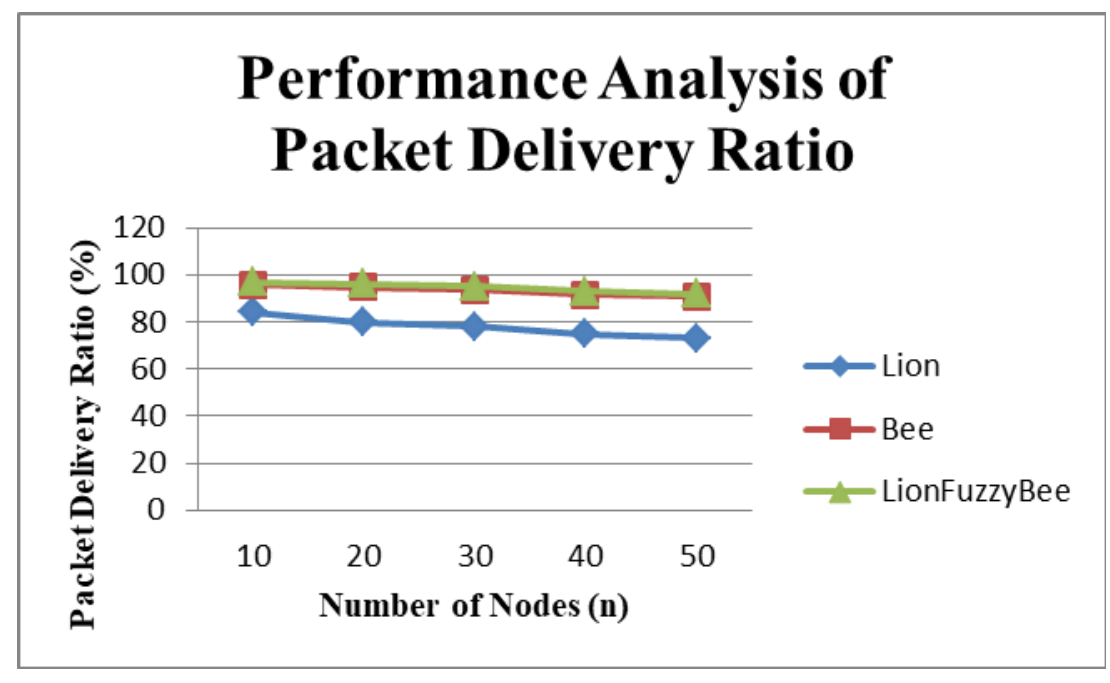

Fig 7: Evaluation Output of Packet Delivery Ratio

Detection Accuracy is the major parameter for analysing congestion detection behaviour of LionFuzzyBee because it correctly predicts the how many number of nodes will be affected by congestion in future using optimised fitness value. Hence it produces highest detection accuracy rate and the result is illustrated in Fig.8 


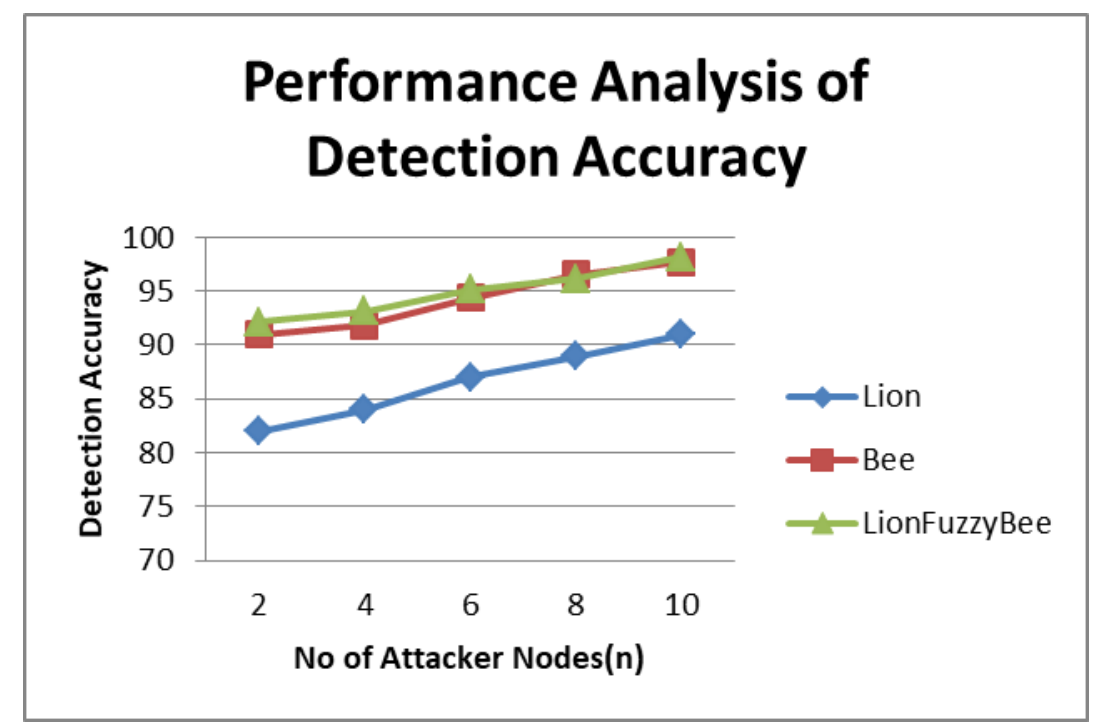

Fig 8: Evaluation Output of Detection Accuracy

Throughput is one of important parameter for identifying congestion because it measures the packet size along with delay. The LionFuzzyBee approach increase the throughput and the result is illustrated in Fig.9. It greatly reduced the congestion behaviour of WSN.

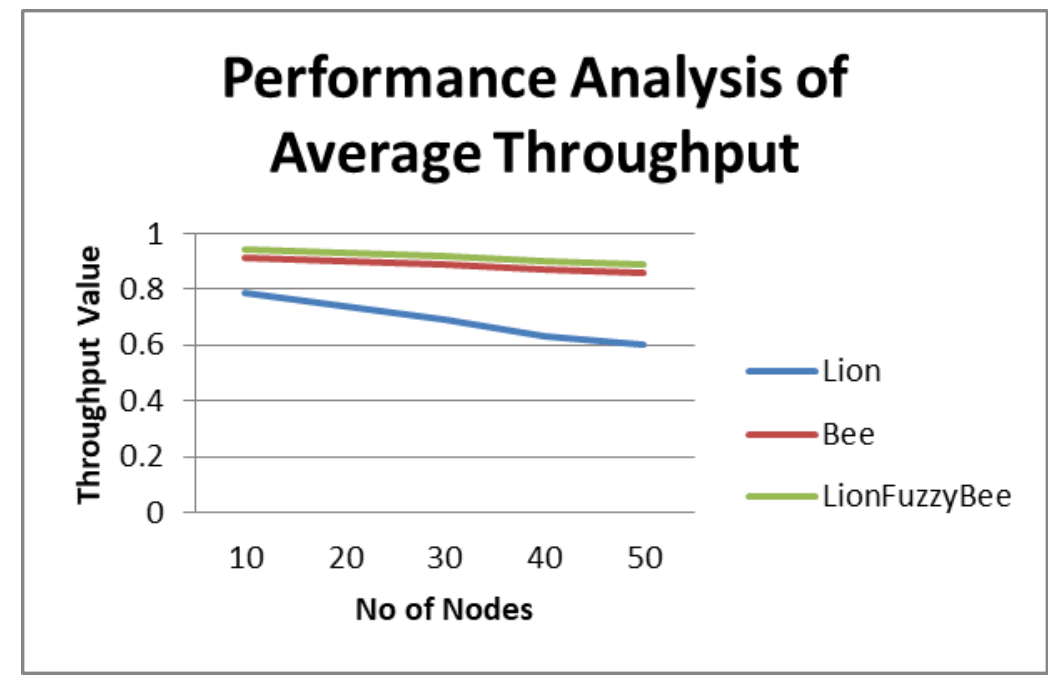

Fig 9: Evaluation Output of Average Throughput

Energy ratio is one of important parameter for identifying congestion because it finds the remaining energy of each node LionFuzzyBee approach reduces the energy loss through controlling congestion and the result is illustrated in Fig.10 


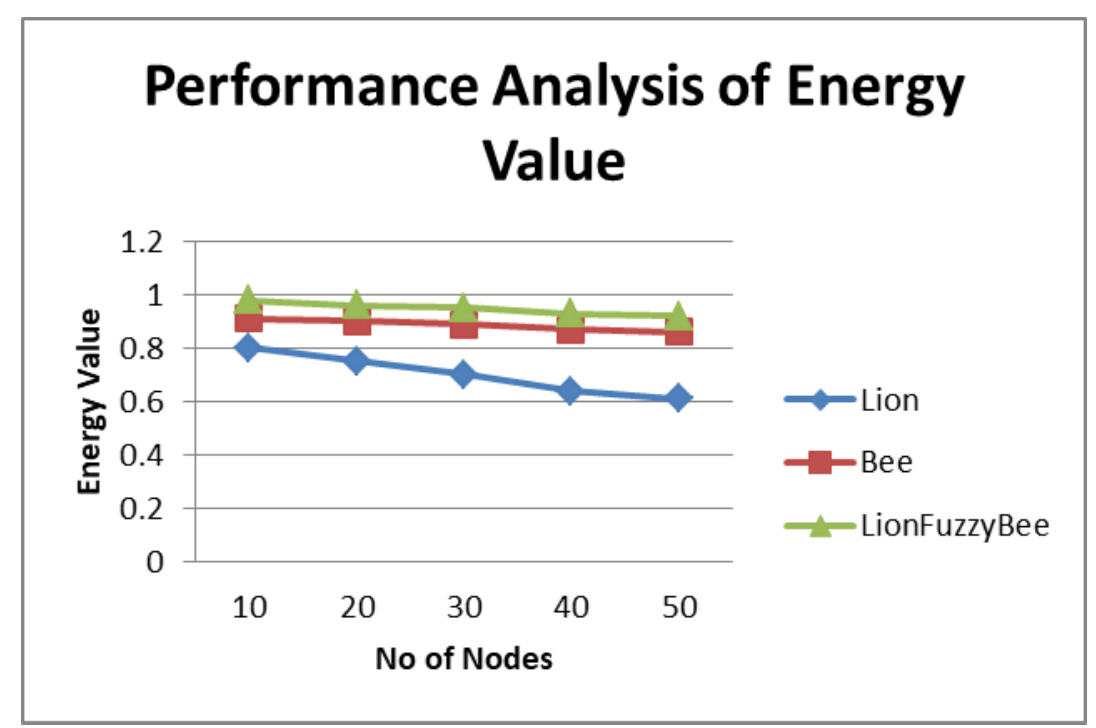

Fig 10: Evaluation Output of Energy Value

Packet loss ratio is one of important parameter for identifying congestion because it counts the number of transmitted packet. When Congestion occurs, it leads to packet loss. But LionFuzzyBee approach decreases the packet loss through controlling congestion and the result is illustrated in Fig. 11.

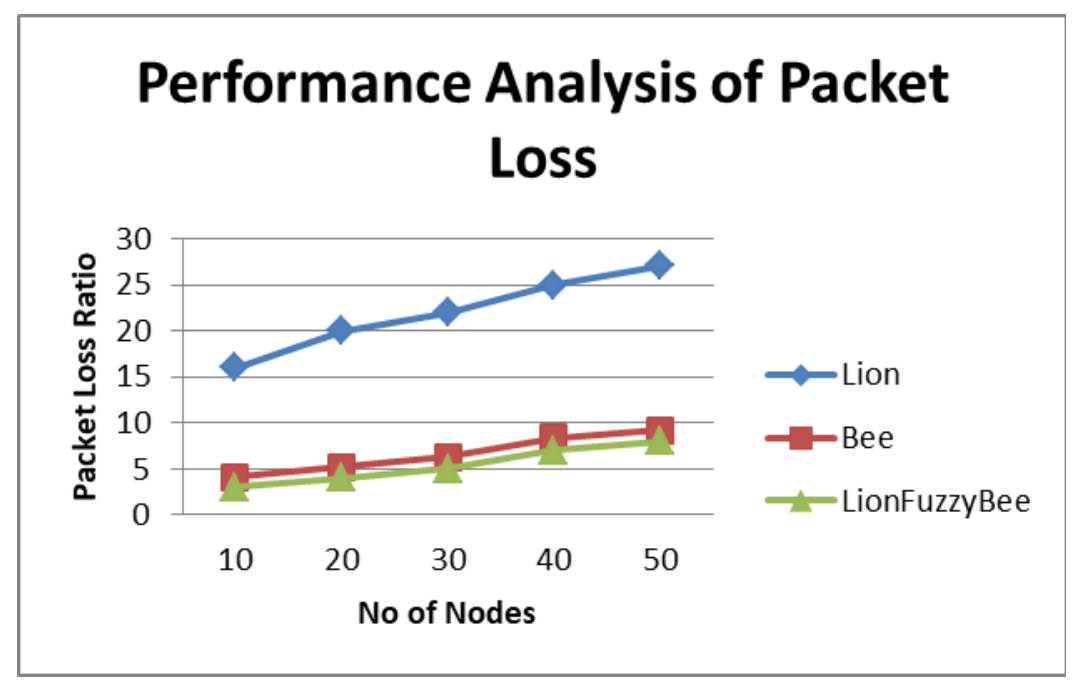

Fig 11: Evaluation Output of Packet Loss

Network Lifetime is one of important parameter for identifying congestion because it finds the remaining energy of each node. Congestion leads to energy loss. It lead to decrease the network lifetime. But this approach increase the network lifetime by reduces the energy loss. Hence it produces highest network lifetime value for all nodes. To prove that, The proposed LionFuzzyBee based congestion control approach's network lifetime value is compared with Lion and Bee based congestion control approaches through varying the number of nodes from 10 to 50 and the result is illustrated in Fig.12. The network lifetime of LionFuzzyBee have higher value than Lion and Bee. 


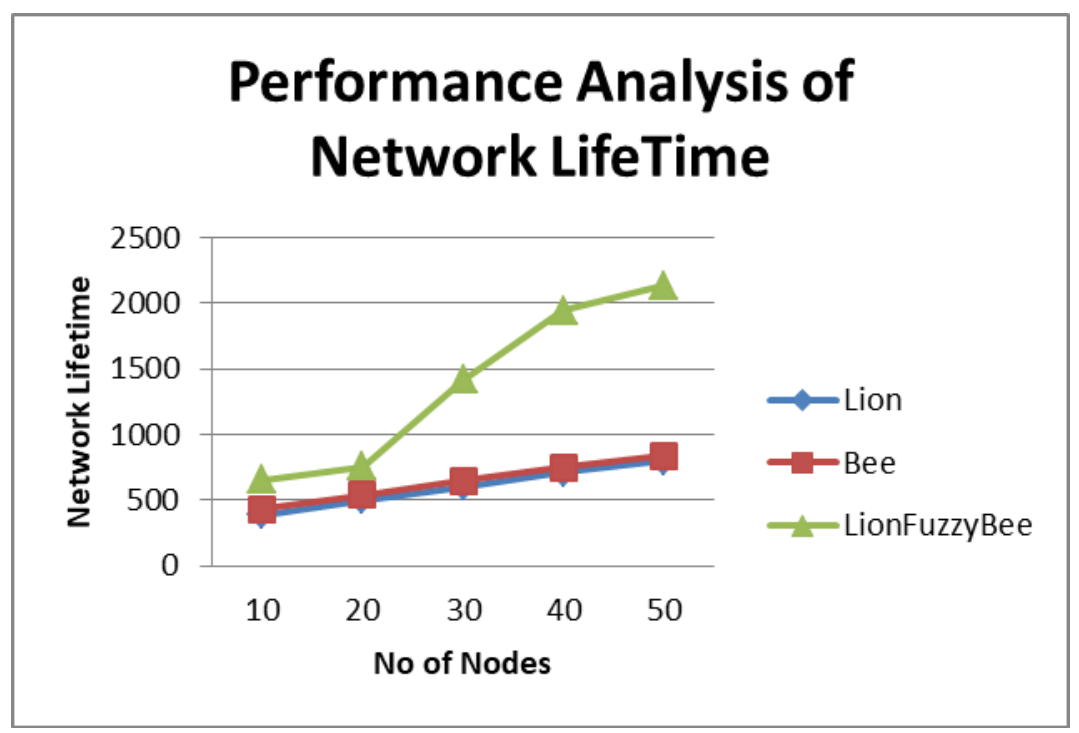

Fig 12: Evaluation Output of Network Lifetime

\section{Conclusion}

This work introduced a new hybrid bio congestion control system called the LionFuzzyBee method. This presented system is compared to a lion and a bee optimizations. The metrics used for comparison are average throughput, packet delivery ratio, detection accuracy, energy value, network lifetime, packet loss. Comparison output specifies that the LionFuzzyBee provides superior output than the Lion and Bee. In this way, the difficulty of congestion in WSN is solved through LionFuzzyBee algorithm.

\section{References}

[1] Hafiza Syeda Zainab Kazmi ,Nadeem Javaid ,Muhammad Imran, Fatma Outay , "Congestion control in wireless sensor networks based on support vector machine, grey wolf optimization and differential evolution” IEEE International Conference 2019.

[2] Karishma Singh, Karan Singh,Le Hoang Son,Ahmed Aziz, "Congestion control in wireless sensor networks by hybrid multi-objective optimization algorithm," Computer. Networks, vol.138, pp. 90-107, 2018

[3] N.T Tam, D.T. Hai,, Le.Hoang. Son, L.T. Vinh (2018). Improving lifetime and network connections of 3D wireless sensor networks based on fuzzy clustering and particle swarm optimization. Wireless Networks, vol.24, pp. 1477-1490, Jul. 2018.

[4] Vaibhav Eknath Narawade, Uttam D. Kolekar, "Congestion Avoidance and Control in Wireless Sensor Networks using Epsilon Constraint based Adaptive Cuckoo Search,” International Education \& Research Journal [IERJ], vol. 3, no.5, May. 2017.

[5] Mukhdeep Singh Manshahia, "Water Wave Optimization Algorithm based Congestion Control and Quality of Service Improvement in Wireless Sensor Networks," Transactions on Networks and Communications, vol. 5, no.4, pp. 31-39, Aug. 2017.

[6] J.Lalitha, Dr. C.Kalaiselvi "Energy Efficient \& Congestion Control in Wireless Sensor Network using Firefly Algorithm," International Journal of Emerging Technology in Computer Science \& Electronics (IJETCSE), vol. 23,no.5, Sep. 2016.

[7] Chandni, Anjali Bharti, Jyoti, "Optimization through Bio Inspired Algorithms in Wireless Sensor Network: Survey and Future Directions,", pp. 243-246, 2015. ISSN ;1694-2310

[8] Raghavendra, Krishna, Znati,“Wireless Sensor Networks”,Springer-Verlag, 2004.

[9] Kemal Akkaya, Mohamed Younis "A survey on routing protocols for wireless sensor networks", AdHoc Networks, pp.325-349, 2005.

[10] I.F. Akyildiz, Weilian Su,Y. Sankarasubramaniam, E. Cayirci,“A survey on sensor networks”, IEEE Communications Magazine,pp.102-114, 2002.

[11] Anu Verma, Nitin Mittal, "Congestion Controlled WSN using Genetic Algorithm with different Source and Sink Mobility Scenarios," International Journal of Computer Applications, vol. 101, no.13, Sep 2014.

[12] Pavlos Antoniou, et al "Congestion control in wireless sensor networks based on bird flocking behavior", Comp Net, vol. 57, No.5,pp. 1167- 1191, Apr. 2013.

[13] Gholipour, M., Haghighat, A. T., \& Meybodi, M. R.Hop-by- Hop Congestion Avoidance in wireless sensor networks based on genetic support vector machine. Neurocomputing, Vol 223, pages63-76,2017.

[14] Abbas Ali Rezaee ,Mohammad Hossein Yaghmaee ,Amir Masoud Rahmani, "Optimized Congestion Management Protocol for HealthcareWireless Sensor Networks", Wireless Personal Communications, vol. 75, . 2014.

[15] [15] Zhu, L., Zhang, Z., \& Xu, C. (2017). Secure data aggregation in wireless sensor networks. In Secure and Privacy-Preserving Data Communication in Internet of Things (pp. 3-31). Springer

[16] Haiming Chen, Li Cui, G Zhou(2017). A Light-Weight Opportunistic Forwarding Protocol with Optimized Preamble Length for LowDutyCycle Wireless Sensor Networks. Journal of Computer Science and Technology, 32(1), 168-180.

[17] Dervis Karaboga, "Artificial Bee Colony Algorithm”, 2010, Scholarpedia, 5(3):6915.

[18] B.R.Rajakumar, "The Lion's Algorithm:A New Nature-Inspired Search Algorithm”, Procedia Technology, Volume 6, 126-135, 2012.

[19] Bo Wang, XiaoPing Jin, Bo Cheng, "Lion Pride Optimizer:An Optimization Algorithm Inspired By Lion Pride Behavior", Springer, 2369-2389(2012)

[20] S. Misra, V. Tiwari, and M. Obaidat, "(LACAS): learning automata based congestion avoidance scheme for healthcare wireless sensor networks," IEEE Journal on Selected Areas in Communications vol. 27, no. 4, May 2009, pp. $466-479$. 
[21] Amir Hossein Gandomi et al. "Bat algorithm for constrained optimization tasks," Neural Computing and Applications, 2013, 22(6), pp: $1239-1255$

[22] AntonioM Ortiz,et al. "Fuzzy-logic based routing for dense wireless sensor networks," Telecommunication Systems,2013, 52(4), pp: 2687- 2697.

[23] Adis Alihodzic, and Milan Tuba, "Improved bat algorithm applied to multilevel image thresholding," The Scientific World Journal, 2014 (2014).

[24] Salvador Climent, Juan Vicente Capella, Nirvana Meratnia and Juan Jos Serrano, "Underwater sensor networks: A new energy efficient and robust architecture," Sensors, 2012, 12(1), pp: 704-731

[25] Seokhoon Yoon, Abul K. Azad, Hoon Oh and Sunghwan Kim, "Aurp: An auv-aided underwater routing protocol for underwater acoustic sensor networks, "Sensors 2012, 12(2), pp:1827-1845.

[26] Sheng-Shih Wang and Ze-Ping Chen, "LCM: a link-aware clustering mechanism for energy-efficient routing in wireless sensor networks, " Sensors Journal, IEEE, 2013, 13(2) pp: 728-736.

[27] Selim Yilmaz and Ecir U. Kucuksille, "Improved bat algorithm (IBA) on continuous optimization problems," Lecture Notes on Software Engineering, 2013,1(3), pp: 279-283.

[28] MiaoZhao, Ji Li, and Yuanyuan Yang, "A framework of joint mobile energy replenishment and data gathering in wireless rechargeable sensor networks," IEEE Transactions on Mobile Computing, 2014, 13(12), pp: 2689-2705.

[29] Xin-She Yang and Amir H Gandomi, "Bat algorithm: a novel approach for global engineering optimization," Engineering Computations, 2012, 29(5), pp: 464-483.

[30] Miguel Garcia et al. "Saving energy and improving communications using cooperative group-based wireless sensor networks," Telecommunication Systems, 2013, 52, pp: 2489-2502.

[31] Chieh-Yih Wan, Shane B. Eisenman, and Andrew T. Campbell,"CODA: Congestion detection and avoidance in sensor networks." Proceedings of the 1st international conference on Embedded networked sensor systems. ACM, 2003.

[32] C. Caione, D. Brunelli, and L. Benini, "Distributed compressive sampling for lifetime optimization in dense wireless sensor networks," IEEE Transactions on Industrial Informatics, vol. 8, no. 1,2012.

[33] X. Wang and K. Kar, "Distributed algorithms for max-min fair rate allocation," in ALOHA networks, 2004.

[34] Jaewon Kang, Yanyong Zhang, and Badri Nath. "TARA: topology-aware resource adaptation to alleviate congestion in sensor networks." IEEE Transactions on Parallel and Distributed Systems 18,7 (2007): 919-931.

[35] Liqiang Tao, Fengqi Yu "ECODA: enhanced congestion detection and avoidance for multiple class of traffic in sensor networks." IEEE transactions on consumer electronics $56.3(2010)$.

[36] Nazmul Siddique, Hojjat Adeli "Nature Inspired Computing: An Overview and Some Future Directions", Cognitive Computation, vol. 7, pp. 706-714, 2015. 